\title{
Effect of bacterial intra-species community interactions on the production and activity of volatile organic compounds
}

\author{
Jianing Wang ${ }^{\dagger}$, Xinlan Mei ${ }^{\dagger}$, Zhong Wei, Waseem Raza*, Qirong Shen \\ Jiangsu Provincial Key Lab for Organic Solid Waste Utilization, National Engineering Research Center for Organic-based Fertilizers, Jiangsu \\ Collaborative Innovation Center for Solid Organic Waste Resource Utilization, Nanjing Agricultural University, Nanjing 210095, China
}

\section{ARTICLE INFO}

\section{Article history:}

Received March 31, 2020

Revised June 9, 2020

Accepted July 15, 2020

\section{Keywords:}

Biodiversity

Community richness

Ecosystem functioning

Intra-species interactions

Plant-microbe interactions

\begin{abstract}
A B S T R A C T
Microorganisms experience intra- and inter-species interactions in the soil, and how these interactions affect the production of microbial volatile organic compounds (VOCs) is still not well-known. Here we evaluated the production and activity of microbial VOCs as driven by bacterial intra-species community interactions. We set up bacterial communities of increasing biodiversity out of 1-4 strains each of the Gram-positive Bacillus and Gram-negative Pseudomonas genera. We evaluated the ability of each community to provide two VOCmediated services, pathogen suppression and plant-growth promotion and then correlated these services to the production of VOCs by each community. The results showed that an increase in community richness from 1 to 4 strains of both genera increased VOC-mediated pathogen suppression and plant-growth promotion on agar medium and in the soil, which was positively correlated with the production of pathogen suppressing and plant growth-promoting VOCs. Pseudomonas strains maintained while Bacillus strains reduced community productivity with an increase in community richness and produced eight novel VOCs compared with the monocultures. These results revealed that intra-species interactions may vary between Gram-negative and Gram-positive species but improved VOC-mediated functioning with respect to pathogen suppression and plant-growth promotion by affecting the amount and diversity of produced VOCs potentially affecting plant disease outcomes.
\end{abstract}

(c) Higher Education Press 2020

\section{Introduction}

Plant roots are associated with a dense multispecies community that provides multiple benefits to plants, including plant growth stimulation and pathogen suppression (Mendes et al., 2015). The rhizosphere microbiome is often approached as the community of microbes in direct contact with plant host, mediated by diffusible molecules and contact-mediated

\footnotetext{
* Corresponding author

E-mail address: waseem@njau.edu.cn (W. Raza)

†Jianing Wang and Xinlan Mei equally contributed to this manuscript.
}

interactions (Westhoff et al., 2017). Recent studies have demonstrated that volatile organic compounds (VOCs) produced by microorganisms can have a deep impact on a range of organisms, including plants and pathogens (Schmidt et al., 2015). The VOCs are low molecular weight and high vapor pressure lipophilic compounds, and are generally produced by microbes through catabolic pathways (Schulz and Dickschat, 2007). Microbial VOCs have been reported for their antibacterial, antifungal, nematocidal activities, and cellto-cell communication (Gu et al., 2007; Raza et al., 2016). In addition, VOCs not only improve plant growth but also induce plant defense against abiotic and biotic stresses (Kanchiswamy et al., 2015). Compared to other microbial compounds, VOCs can show an effect on longer distances far from the 
production point through the atmosphere and porous soils because of their diffusibility (Leff and Fierer, 2008). This implies that plant growth and health may be under the direct influence of a much bigger microbial community than originally expected (Tahir et al., 2017).

In the rhizosphere, the functionality and effects of the microbiome on plant fitness are directly determined by the species composition and biodiversity. Different species interact, sometimes, leading to death, generating new metabolic pathways or expressing silent secondary metabolites gene clusters (Jurburg and Salles, 2015; Hu et al., 2017). Such effects may also occur without contact via the production of VOCs (Garbeva et al., 2011). Most studies involving VOCmediated interactions have been conducted using a single microorganism. Recently, many studies regarding communication among different soil bacterial species have been conducted, but such reports about the production and activity of VOCs in a mixed community are scarce. Schulz-Bohm et al. (2015) reported that a mixture of five phylogenetically different bacterial strains produced a different blend of VOCs compared to the monoculture. Alternatively, Tyc et al. (2015) did not find novel or new VOCs in a pairwise comparison of phylogenetically different bacterial species, but only the combination of VOCs produced as a monoculture. Recently, the effects of inter-species interactions on the production and activity of plant growth-promoting and pathogen inhibiting VOCs were explored (Raza et al., 2020). Different microbial species display different ecological characteristics in response to the presence of other similar or different species, so the cumulative outcome may change compared to individual species (Loreau and Hector, 2001). In this context, how intra-species interactions mediate the production and functioning of VOCs is still unknown. Here we tried further extending the knowledge of VOC-mediated functioning driven by bacterial intra-species community interactions. In our study system, we separately set up experimental bacterial communities of increasing biodiversity out of $1-4$ strains each welldescribed Gram-positive and Gram-negative soil bacterial species Pseudomonas fluorescence and Bacillus amyloliquefaciens, respectively. We assessed the ability of each community to provide two microbiome services, pathogen suppression (Ralstonia solanacearum) and plant-growth promotion (Arabidopsis thaliana), without direct contact. We then related these two services to the amount and diversity of the VOC cocktails produced by each community.

\section{Materials and methods}

\subsection{Bacterial strains and assemblage of communities}

Four strains each, two bacterial genera Bacillus and Pseudomonas isolated from the rhizosphere of different plants were selected (Table S1). We performed preliminary experiments to ensure that the pathogen suppression and plant-growth promotion by the selected bacterial strains were mediated only by VOCs (Table S1). For the assemblage of bacterial communities, the strains were grown overnight in a nutrient broth at $30^{\circ} \mathrm{C}$, washed twice with $0.85 \% \mathrm{NaCl}$ and adjusted to the cell counts of $1 \times 10^{7}$ colony forming units (CFU) $\mathrm{mL}^{-1}$. The strains of each genus were mixed separately in equal proportion $(500 \mu \mathrm{L}$ ) to obtain 15 combinations (Table S2) with richness levels ranging from monocultures to the mixture of 4 strains considering that at each richness level, each genotype is replicated three times according to Bell et al. (2009). At each richness level, the total cell concentration of the monoculture or mixed cultures was $1 \times 10^{7} \mathrm{CFU} \mathrm{mL}{ }^{-1}$. The target pathogen used was Ralstonia solanacearum QLRs-1115 [RS; China General Microbiology Culture Collection Center accession No. 9487; Wei et al., 2011]. During the experiments, RS was grown overnight in nutrient medium at $30^{\circ} \mathrm{C}$, washed twice and adjusted to the cell counts of $1 \times 10^{7}$ CFU $\mathrm{mL}^{-1}$ with $0.85 \% \mathrm{NaCl}$. The bacterial strains and pathogenic strain were stored at $-80^{\circ} \mathrm{C}$ in nutrient medium containing $70 \%$ glycerol.

2.2 Growth assay of bacterial monocultures and assembled communities

To determine the effect of intra-species interactions on the growth of bacterial communities, each well of the microtiter plates was filled with $195 \mu \mathrm{L}$ minimal salt medium (Harwood and Cutting, 1990) modified with $0.4 \%$ tryptone soy broth (w/v) and $1.5 \%$ sucrose, and inoculated separately with $5 \mu \mathrm{L}$ cell suspension of each bacterial community of both genera, prepared as described above (Table S2). The growth of Pseudomonas and Bacillus strains was determined by serial plating on nutrient agar medium after $36 \mathrm{~h}$ at $30^{\circ} \mathrm{C}$ and 170 $\mathrm{r} \min ^{-1}$ and was represented as community productivity at different bacterial richness levels. The experiment was repeated twice in triplicate.

2.3 VOC-mediated pathogen suppression and plant-growth promotion assays

For VOC-mediated pathogen suppression by bacterial communities on agar medium, the cell suspension of bacterial strains was prepared separately as described above, at each richness level, was spotted at 2 points $5 \mathrm{~cm}$ apart ( $5 \mu \mathrm{L}$ each) onto one compartment of the divided Petri plates (85-mm diameter) containing modified minimal salt agar medium prepared as described above except with $1.5 \%$ agar. While the cell suspension of RS was spot-inoculated $5 \mathrm{~cm}$ apart at 2 points onto the other compartment of the divided Petri plates containing nutrient agar medium. The plates were placed overnight at $30^{\circ} \mathrm{C}$ to initiate bacterial growth and then sealed with Parafilm and incubated at $30^{\circ} \mathrm{C}$ for $3 \mathrm{~d}$ further. The Petri plates only inoculated with RS were used as a control. After $3 \mathrm{~d}$, the RS colonies were removed along with the agar medium using a sterilized scalpel, suspended $(1 \mathrm{~mL})$ and diluted (500 times) using sterilized water and spread on nutrient agar plates. The CFU mL ${ }^{-1}$ of RS was determined after incubation for $2 \mathrm{~d}$ at $30^{\circ} \mathrm{C}$. The experiment was repeated twice in triplicate. As evaluated in a previous experiment, the 
growth of used Bacillus and Pseudomonas strains was not affected by the VOCs of RS (Raza et al., 2020).

For VOC-mediated pathogen-suppression assay in soil, the healthy soil was obtained from Yixing, China [for soil properties, see our previous report (Raza et al., 2015)]. The soil was sterilized at $121^{\circ} \mathrm{C}$ for $60 \mathrm{~min}$ and after cooling to room temperature, $7.0 \mathrm{~g}$ (dry weight) soil was first mixed with the cell suspensions of bacterial communities $(1 \mathrm{~mL})$, prepared separately as described above, for each richness level and then added to one compartment of the divided Petri plates except the controls where the soil was added without bacterial inoculation. The other compartment of the Petri plate was spot-inoculated with pathogen RS onto nutrient agar medium as described above. The plates were placed overnight at $30^{\circ} \mathrm{C}$ and then sealed with Parafilm and incubated again for $3 \mathrm{~d}$. The CFU mL ${ }^{-1}$ of RS was determined as described above and the VOC effects were presented as the percent increase or decrease in pathogen suppression relative to the control treatment. The experiment was repeated twice in triplicate.

For VOC-mediated plant-growth promotion assay by bacterial communities, the same methodology was used, except for the spot inoculation of RS, three seedlings of $A$. thaliana (Col-0) were transferred to one compartment of the divided Petri plates containing half-strength Murashige and Skoog (MS) agar medium (0.8\% agar, $\mathrm{pH} 5.7)$. Before transfer, the surface-sterilized seeds were first vernalized for $2 \mathrm{~d}$ at $4^{\circ} \mathrm{C}$ and then placed in a growth chamber for $4 \mathrm{~d}\left(22^{\circ} \mathrm{C}\right.$ temperatures, $12 \mathrm{~h}$ light, $12 \mathrm{~h}$ dark, $40 \mathrm{~W}$ fluorescent light). On the other compartment of the Petri plates, two spot inoculations of bacterial strains at different richness levels on the modified minimal salt agar medium and in the soil were performed as described above. The Petri plates without bacterial inoculation were used as controls. Two weeks later, the whole plant was removed and the fresh weight $(\mathrm{mg}$ plant $^{-1}$ ) was determined after washing plant with sterilized water and blot-drying. The VOC effects were presented as the percentage increase or decrease in plant growth relative to the control treatment and the experiment was repeated twice in triplicate.

2.4 VOC analysis using gas chromatography-mass spectroscopy (GC-MS)

To analyze the VOC profiles produced by all bacterial strains and communities, cell suspensions $\left(1 \times 10^{7} \mathrm{CFU} \mathrm{mL}^{-1}\right)$ were prepared as described above and 2 spots ( $5 \mu \mathrm{L}$ each) inoculated on minimal salt agar medium (same as above) in a $100 \mathrm{~mL}$ vial was prepared in triplicate and placed at a temperature of $30^{\circ} \mathrm{C}$. After overnight growth, the vials were sealed and incubated at $30^{\circ} \mathrm{C}$ for $3 \mathrm{~d}$. The vials without the inoculation of bacteria were used as controls. After incubation, $5 \mu \mathrm{L}$ of (Z)-3-hexenyl acetate (1 $\mathrm{mM}$ ) as an internal standard was added to the vials. Then, the extraction of VOCs using solid-phase microextraction (SPME) fiber and GC-MS analysis was performed as reported in our previous reports (Raza et al., 2015, 2016, 2020). The VOC was considered as identified if it had a match factor greater than 800 , contained all main peaks of the listed compound, and its retention index difference was not larger than five with the listed compound (for a semi-standard non-polar column). Except for 12 unidentified and 2 commercially unavailable VOCs, the production of identified VOCs was further confirmed by comparing them with standard compounds purchased from Sigma; Tokyo Chemical Industry Co., Ltd. Japan and Aladdin Reagent Database, Inc., Shanghai, China. The standards were analyzed similarly as those using SPME fiber. As an indirect approach to estimate the relative amount (concentration) of each VOC, the number of VOCs (number of peaks) produced in each treatment were recorded and the chromatographic peak area was expressed in arbitrary units (a.u.) relative to the peak area of (Z)-3-hexenyl acetate (internal standard).

\subsection{Classification of VOCs into pathogen suppressing and plant growth-promoting compounds}

Except for the 12 unidentified and 2 commercially unavailable VOCs, all VOCs were assessed for their pathogen suppression and plant growth-promoting activity. For pathogensuppression assay, the cell suspension of RS prepared as described above was spot-inoculated at two points onto one compartment of the divided Petri plates containing nutrient agar medium and incubated overnight at $30^{\circ} \mathrm{C}$. Later, the other half of the Petri plates were separately inoculated with $30 \mu \mathrm{L}$ of solutions of pure VOCs $(10,50,250,1$, and 5 $\mu \mathrm{g} \mathrm{mL}^{-1}$ ) in methanol on an approximately $10 \mathrm{~mm}$ (diameter) sterile filter paper disk (Whatman filter paper, $6 \mu \mathrm{m}$ pore size) to give a final amount of $0.3,1.5,7.5,30$, and $150 \mu \mathrm{g}$ final amount of each pure $\mathrm{VOC}$, respectively. Petri plates were sealed with Parafilm and incubated at $30^{\circ} \mathrm{C}$ for $3 \mathrm{~d}$. Later, the $\mathrm{CFU} \mathrm{mL} \mathrm{m}^{-1}$ of $\mathrm{RS}$ was determined and expressed as described above.

For the plant-growth promotion assay, the same methodology was used, except for the spot inoculation of RS, three Arabidopsis seedlings were placed onto the other half of the Petri plates containing half-strength MS agar medium. The plates were sealed with Parafilm and after two weeks, the fresh weight of the plant was determined and expressed as described above. For pathogen suppression and plant-growth promotion, the sterile filter paper disks inoculated with nothing or with methanol were used as control treatments (both control treatments did not show any difference). The experiment was repeated twice in triplicate.

\subsection{Statistical analysis}

The differences in bacterial communities, strains, and genera were analyzed using ANOVA and Tukey's tests. Regression analysis was used to analyze the relationship between bacterial community richness and VOC-mediated pathogen suppression and plant-growth promotion, the relative amount of VOCs, number of VOCs, and total community growth (cell counts $\mathrm{mL}^{-1}$ ). The identity effect of bacterial strains was analyzed by performing a separate generalized linear model 
(GLM) expressing pathogen suppression and plant-growth promotion as a function of the presence of each bacterial strain (based on binary predictors). Statistical analyses were conducted with SPSS version 19.0 statistical software (SPSS, Inc., Chicago, IL, USA).

\section{Results and discussion}

3.1 Effect of monocultures and community richness on VOCmediated pathogen suppression and plant-growth promotion

In this study, all eight tested bacterial strains showed VOCmediated pathogen suppression and plant-growth promotion. Among different Pseudomonas strains, Q8r1-96 showed the highest VOC-mediated pathogen suppression both on agar medium $\left(\mathrm{F}_{3,8}=52.73, P<0.0001\right)$ and in the soil $\left(\mathrm{F}_{3,8}=155.7\right.$, $P<0.0001$; Fig. S1A). Among the Bacillus strains, NJN-6 showed the highest VOC-mediated pathogen suppression on agar medium $\left(\mathrm{F}_{3,8}=187.4, P<0.0001\right)$, and strain SQR-9 was the most effective in soil $\left(F_{3,8}=207.3, P<0.0001\right.$; Fig. S1B). For VOC-mediated plant-growth promotion, among the Pseudomonas strains, Q2-87 showed the highest plantgrowth promotion both on agar medium $\left(F_{3,8}=69.53\right.$, $P<0.0001)$ and in soil $\left(\mathrm{F}_{3,8}=158.5, P<0.0001\right.$; Fig. $\left.\mathrm{S} 1 \mathrm{C}\right)$. Among the Bacillus strains, strain NJN-6 exhibited highest plant-growth promotion both on agar medium $\left(\mathrm{F}_{3,8}=162.9\right.$, $P<0.0001)$ and in soil $\left(\mathrm{F}_{3,8}=298.2, P<0.0001\right.$; Fig. S1D). Except for VOC-mediated pathogen-suppression activity of Bacillus strains, the most effective strain of both Pseudomonas and Bacillus genera on agar medium was also the most effective in the soil. However, some strains showed differences in their VOC-mediated effects on agar medium and in soil (Fig. S1), which might be related to the differences in nutrient composition of agar medium and soil as VOC production can vary depending on the substrate composition (Lazazzara et al., 2017)

The results of the bacterial community richness and VOCmediated activity revealed that the increase in community richness of Pseudomonas strains increased pathogen suppression, which on agar medium was $26 \%$ at richness level 1 and $33 \%$ at richness level $4\left(\mathrm{~F}_{3,41}=19.9, P<0.0001\right.$; Fig. $\left.1 \mathrm{~A}\right)$. Similar results were obtained in soil, where $24 \%$ pathogen suppression at richness level 1 rose to $31 \%$ at richness level 4 $\left(F_{3,41}=32.7, P<0.0001\right.$; Fig. S2A $)$. For the Bacillus strains, an increase in community richness levels increased the pathogen suppression from $26 \%$ at richness level 1 to $35 \%$ at richness level 4 on agar medium $\left(\mathrm{F}_{3,41}=27.1, P<0.0001\right.$; Fig $1 \mathrm{~B})$, while in soil, $24 \%$ pathogen suppression at richness level 1 rose to $31 \%$ at richness level $4\left(F_{3,41}=86.9, P<0.0001\right.$; Fig. S2B).

For VOC-mediated plant-growth promotion, the increase in community richness of Pseudomonas strains increased the plant-growth promotion from $65 \%$ at richness level 1 to $109 \%$ at richness level 4 on agar medium $\left(\mathrm{F}_{3,41}=16.1, P<0.0001\right.$; Fig. 1C). In soil, similar results were obtained and an increase in plant-growth promotion by $55 \%$ at richness 1 rose to $90 \%$ at richness level $4\left(\mathrm{~F}_{3,41}=13.7, P=0.001\right.$; Figure $\left.\mathrm{S} 2 \mathrm{C}\right)$. For Bacillus strains, plant-growth promotion of $43 \%$ at richness level 1 rose to $114 \%$ at richness level 4 on agar medium $\left(F_{3,41}\right.$ $=13.8, P<0.0001$; Fig. 1D), while in soil, the increase in plantgrowth promotion by $48 \%$ at richness level 1 rose to $88 \%$ at richness level $4\left(\mathrm{~F}_{3,41}=23.9, P<0.0001\right.$; Fig. S2D). The VOCmediated effects observed on the agar medium and in soil showed some differences; however, in general, both showed a significant positive correlation (Fig. S3), which recommends that VOCs produced on agar medium provides a realistic estimate of VOC-mediated activity in the soil.

These results revealed that intra-species interactions within a community improved the VOC-mediated activity with respect to pathogen suppression and plant-growth promotion. Our outcomes are in agreement with several studies that reported positive relationships between the microbial community and plant performance. For example, Wagg et al. (2011) and $\mathrm{Hu}$ et al. (2017) reported that the microbial diversity of communities containing four and eight microbes, respectively, showed positive relationships with plant performance. Alternatively, Jousset et al. (2011) reported a humpshaped association between toxin production and bacterial community richness. In another report, while the VOCmediated pathogen suppression increased, VOC-mediated plant-growth promotion decreased with an increase in bacterial community richness up to richness level 4 (Raza et al., 2020). These differences in results may be related to the use of different bacterial strains compared with those in our study.

3.2 Bacterial strains identity effect on VOC-mediated pathogen suppression and plant-growth promotion

The results of the strain identity effect revealed that among the Pseudomonas strains, Q8r1-96 was the most effective strain and played a significant role in VOC-mediated pathogen suppression and plant-growth promotion both on agar medium and in soil (Fig. S4A). The strain Q2-87 showed a significant contribution only on VOC-mediated plant-growth promotion and strain $\mathrm{Phl} 1 \mathrm{c} 2$ showed a significant contribution only on VOC-mediated pathogen suppression on agar medium. Further confirmation was obtained from the GLM analysis showing that except for strain Q2-87 for pathogen suppression and strain Phl1c2 for plant-growth promotion, all other strains showed significant contribution (Table S3). Among the Bacillus strains, SQR-9 was the most effective strain, which displayed a significant role in VOC-mediated pathogen suppression and plant-growth promotion both on agar medium and in soil (Fig. S4B). The strain NJN-6 showed significant contribution only in VOC-mediated plant-growth promotion and strain T-5 showed a significant contribution only in VOC-mediated pathogen suppression. GLM analysis further revealed that except for strains NJN-6 and FZB42 for pathogen suppression and strain T-5 for plant-growth promotion, all other strains showed a significant contribution. 

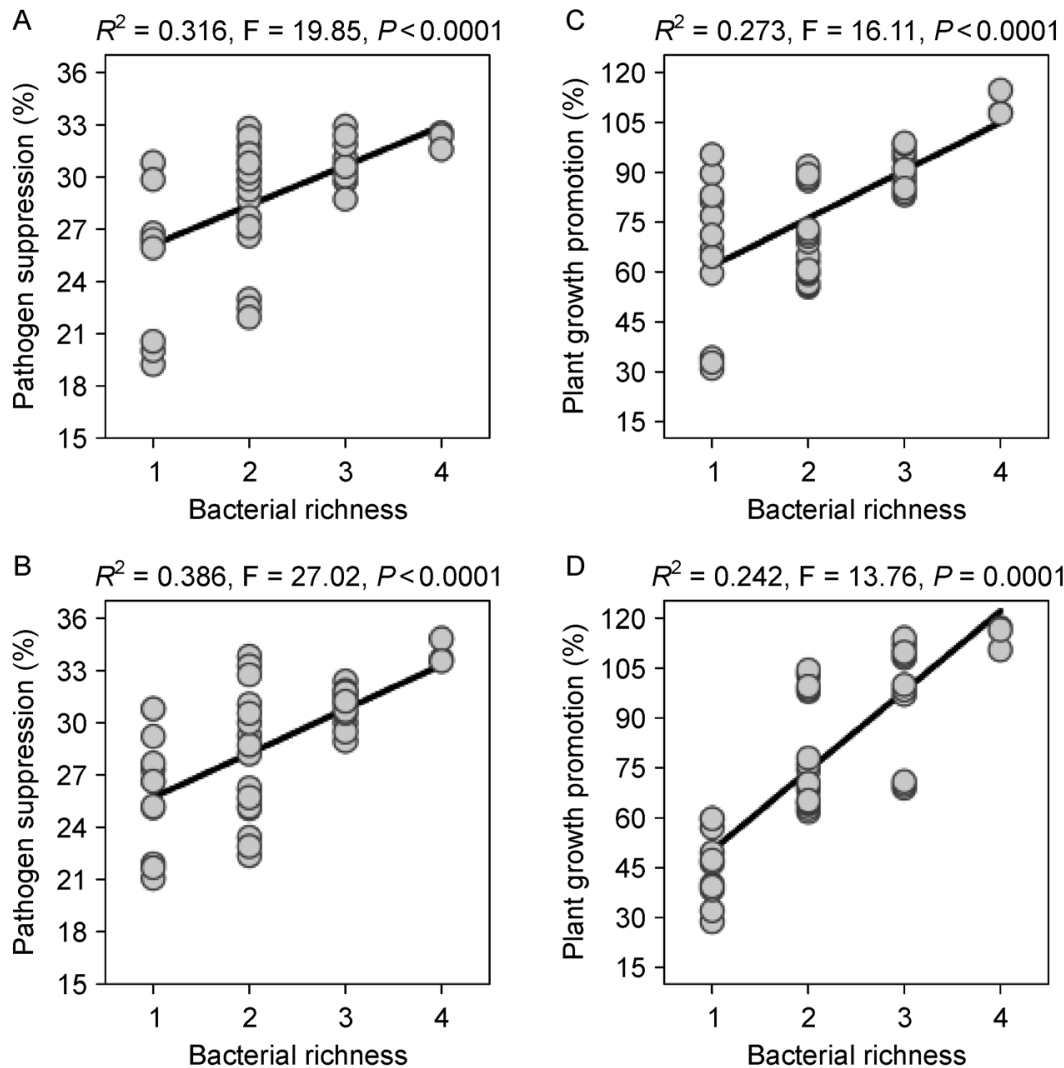

Fig. 1 Volatile organic compounds (VOCs)-mediated pathogen suppression and plant-growth promotion at different bacterial community richness levels on agar medium. Left panels show the pathogen suppression $(A)$ and plant-growth promotion (B) by the VOCs produced by Pseudomonas strains and right panels show pathogen suppression (C) and plant-growth promotion (D) by the VOCs produced by Bacillus strains at different community richness levels. The pathogen suppression was determined as cell counts $\mathrm{mL}^{-1}$ after exposure to the VOCs produced by each bacterial community and expressed as a percentage decrease compared to the control (no VOC exposure). The plant-growth promotion was determined as plant fresh weight (mg) after exposure to the VOCs produced by each bacterial community and expressed as a percentage increase compared to the control. Each data point represents each replicate.

Moreover, the strain identity effect appeared to be more prominent for both the Pseudomonas and Bacillus genera compared to the community richness effect (Table S3). Similar results were reported by Jousset et al. (2014), who showed a significant strain identity effect of $P$. fluorescence PF- 5 as its presence strongly increased the concentration of antibiotics at each level of diversity. Similarly, Hazard et al. (2017) reported that the intraspecific identity of Ectomycorrhiza fungi becomes more important than richness in modulating plant and fungal performance. Alternatively, Hu et al. (2017) revealed that the identity effect of Pseudomonas strains was significant to siderophore production and phosphate solubilization; however, generally, the diversity effects were stronger than the strain identity effects. Bacteria from complex communities of competing and cooperating organisms differ in functionality with both the identity of the present species and their interactions (Becker et al., 2012). In this study, while all strains were playing their role, some strains were dominated the community for VOC production and functioning.
3.3 Effect of monocultures and community richness on the production of pathogen suppressing and plant growthpromoting VOCs

Among different Pseudomonas strains, PF-5 showed the highest relative amount $\left(\mathrm{F}_{3,8}=359.4, P<0.0001\right)$ and number $\left(F_{3,8}=235.7 .2, P<0.0001\right)$ of all VOCs (Fig. S5A). While the highest relative amount $\left(F_{3,8}=343.4, P<0.0001\right)$ and numbers $\left(F_{3,8}=13.33, P=0.015\right)$ of pathogen-suppressing VOCs were produced by strains PF-5 and Q8r1-96. For plant growth-promoting VOCs, while the highest relative amount of VOCs $\left(F_{3,8}=57.26, P=0.001\right)$ was produced by strains Phl1C2 and PF5, the highest number $\left(F_{3,8}=298.2\right.$, $P<0.0001$ ) of VOCs was produced by strain PF-5 (Fig. $\mathrm{S} 5 \mathrm{~B})$. Among the Bacillus strains, SQR-9 showed the highest relative amount $\left(F_{3,8}=655.5, P<0.0001\right.$ for all VOCs, $F_{3,8}=$ 581.6, $P<0.0001$ for pathogen-suppressing VOCs) and numbers $\left(F_{3,8}=64.5, P=0.001\right.$ for all VOCs, $F_{3,8}=64.6, P$ $=0.001$ for pathogen-suppressing VOCs) of all VOCs and 
pathogen-suppressing VOCs (Fig. S5C), while strain FZB42 showed the highest number $\left(F_{3,8}=298.2, P<0.0001\right)$ and strain SQR-9 showed the highest relative amount $\left(F_{3,8}=\right.$ 307.4, $P<0.0001$ ) of plant growth-promoting VOCs (Fig. S5D).

We next explored how bacterial community richness affected the production of pathogen suppressing and plant growth-promoting VOCs on agar medium. The results showed that community richness of strains of both genera had a positive effect on the production of pathogen suppressing and plant growth-promoting VOCs. For Pseudomonas strains, an increase in community richness up to level 4 increased the relative amount of pathogen-suppressing VOCs by 2.8 folds $\left(F_{3,41}=44.3, P<0.0001\right)$ and the numbers of pathogens suppressing VOCs by 2.1 folds $\left(F_{3,41}=92.8, P<0.0001\right)$ compared with richness level 1 (Fig. 2A-B). Similarly, an increase in community richness up to level 4 increased the relative amount of plant growth-promoting VOCs by 2.6 folds $\left(F_{3,41}=117.2, P<0.0001\right)$ and number of plant growthpromoting VOCs by 2.0 folds $\left(F_{3,41}=45.5, P<0.0001\right)$ compared to richness level 1 (Fig. 2C-D).

For the Bacillus strains, an increase in community richness up to level 4 increased the relative amount of pathogensuppressing VOCs by 2.4 folds $\left(F_{3,41}=29.4, P<0.0001\right)$ and numbers of pathogen-suppressing VOCs by 1.6 folds $\left(F_{3,41}=\right.$ 20.7, $P<0.0001)$ compared to richness level 1 (Fig. $2 \mathrm{E}-\mathrm{F}$ ).
Similarly, an increase in community richness up to level 4 increased the relative amount of plant growth-promoting VOCs by 3.6 folds $\left(F_{3,41}=141.6, P<0.0001\right)$ and number of plant growth-promoting VOCs by 2.3 folds $\left(F_{3,41}=112.1\right.$, $P<0.0001)$ compared to richness level 1 (Fig. 2G-H). While all used strains could variably able to produce both pathogen suppressing and plant growth-promoting VOCs in monocultures; the majority of produced VOCs were pathogensuppressing and the relative amount and numbers of pathogen suppressing and plant growth-promoting VOCs were significantly positively correlated with pathogen suppression and plant-growth promotion, respectively (Figs. S6, S7). These results revealed that intra-species interactions within a community improved the VOC-mediated functioning in terms of pathogen suppression and plant-growth promotion by affecting the amount and diversity of produced VOCs. Our results are in agreement with several studies that reported positive relationships between the microbial community and plant performance (Wagg et al., 2011; Hu et al., 2017). However, Jousset et al. (2011) reported a hump-shaped pattern between toxin production and bacterial community richness. Similarly, in another report, while the production of pathogen-suppressing VOCs increased up to richness level 4 , the production of plant growth-promoting VOCs decreased with an increase in bacterial community richness (Raza et al., 2020).
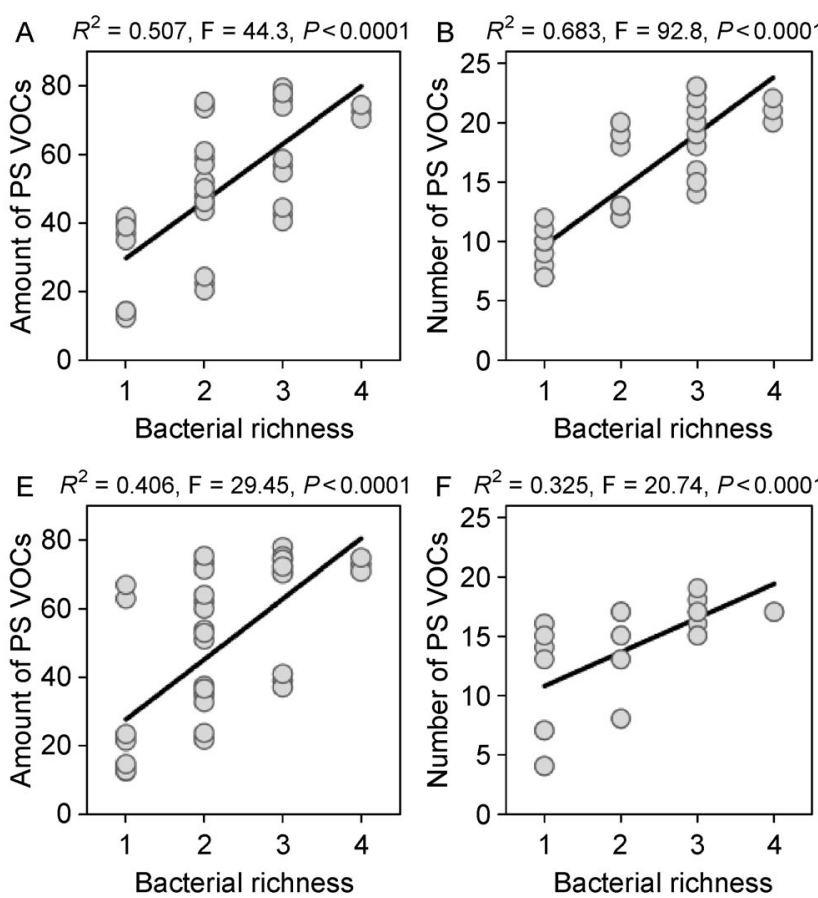
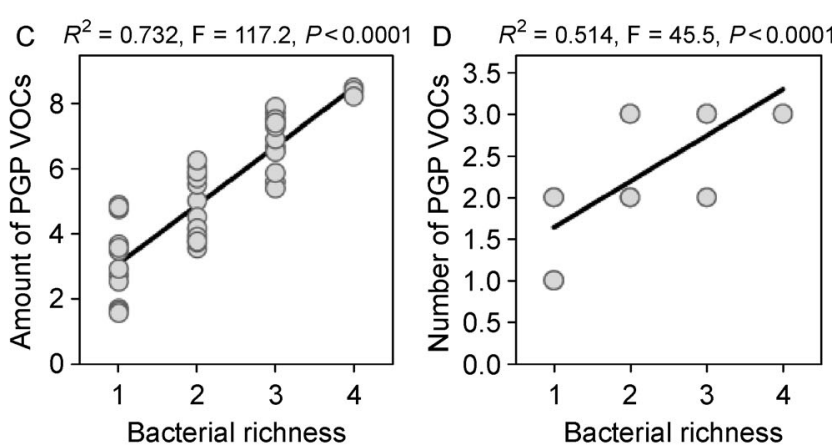

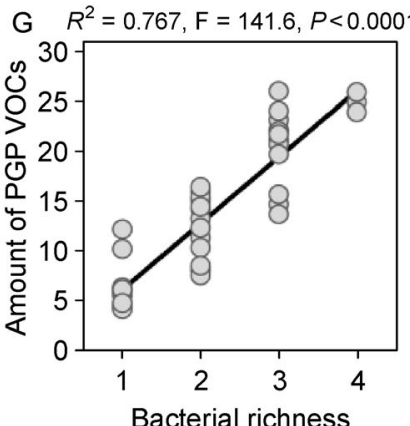

Fig. 2 Volatile organic compounds (VOCs) produced by Pseudomonas fluorescence and Bacillus amyloliquefaciens strains at different community richness levels. The top panel shows the relative amount $(A)$ and number $(B)$ of pathogen-suppressing VOCs and the relative amount (C) and number (D) of plant growth-promoting VOCs produced by Pseudomonas fluorescence strains. The bottom panel shows the relative amount $(E)$ and number $(F)$ of pathogen suppressing VOCs and the relative amount $(G)$ and number $(\mathrm{H})$ of plant growth-promoting VOCs by Bacillus amyloliquefaciens strains. Each data point shows the effect of each replicate. The number of VOCs represents the relative peak area to (Z)-3-hexenyl acetate (internal standard) in arbitrary units (a.u.), as detected by GC-MS analysis. 
3.4 Relationship between pathogen suppression and plant-growth promotion on the production of VOCs

We next investigated whether the production of certain VOCs by bacterial communities could explain the VOC-mediated functioning. For Pseudomonas strains, 45 VOCs were produced and except 3 , all showed significant differences in their production at different bacterial richness levels (Data-Set 1). When VOCs were screened for their activity, 25 (54\%) pathogen-suppressing VOCs were found (Fig. S8A-B). The results of the relative amounts of pathogen-suppressing VOCs at different community richness levels varied. There were 5 VOCs produced at different bacterial richness levels but not at richness level 4 , while one VOC was produced only at richness level 4 . The production of $14 \mathrm{VOCs}$ was highest at richness level 4 , and among those, only 2 VOCs showed significant differences with other richness levels (Data-Set 1). Compared to the monocultures, three new VOCs were produced by Pseudomonas strains at different community richness levels (Fig. S9A) and one of those VOCs showed pathogen suppression activity (Fig. S9B), although it was produced in a relatively small amount (Data-Set 1 ). Except for the two VOCs produced only at richness level 4, one new VOC was produced in all richness levels. There were only three $(6 \%)$ plant growth-promoting VOCs produced at different bacterial community richness levels of Pseudomonas strains (Fig. S8C). Among those, hexadecane was equally produced at all richness levels while heptadecane and tetracosane showed the highest relative amount at richness levels 4 and 2, respectively (Data-Set 1 ). For Bacillus strains, 36 VOCs were produced and except 4 , all showed significant differences in their production at different bacterial richness levels (Data-Set 2). When VOCs were screened for their activity, $23(63 \%)$ VOCs showed pathogen-suppressing activity (Fig. S8A-B). The results of the relative amount of pathogen-suppressing VOCs at different community richness levels varied. There were 5 VOCs produced at different bacterial richness levels but not at richness level 4 , while one VOC was produced only at richness level 4 . The production of 10 VOCs was found highest at richness level 4 , and among those, 5 VOCs showed significant differences with other richness levels (Data-Set 2). Compared with monocultures, 8 new VOCs were produced by Bacillus strains at different community richness levels and 5 of those VOCs showed pathogen-suppressing activity (Fig. S9C-D). Except 2 VOCs, produced only at richness levels 2 and 4 , respectively, other new VOCs were produced at all richness levels (Data-Set 2). There were only three $(9 \%)$ plant growth-promoting VOCs produced at different community richness levels of Bacillus strains (Fig. S8C). Among those, the relative amount of 1,3propanediol was highest at richness level 3 , while the highest production of heptadecane was found at richness level 2. Hexadecane also showed highest production at richness levels 1 and 2 .

Most of the VOCs produced by both Pseudomonas and Bacillus strains showed that pathogen-suppression activity and only three plants growth-promoting VOCs were produced by both genera. The increase in relative amount and number of pathogen suppressing and mainly plant growth-promoting VOCs with the increase in community richness was due to the mixture of VOCs produced by all four bacterial isolates of both genera. Tyc et al. (2015) reported the production of more numbers of VOCs in pairwise combinations, which were the mixture of VOCs of two bacterial isolates; no novel or different VOCs were found. Conversely, Schulz-Bohm et al. (2015) reported that the mixture of five phylogenetically different bacterial strains produced a different blend of VOCs compared to the monocultures. In another study, the emergence of nine new VOCs was found with the increase in community richness as the result of inter-species interactions, including three pathogen-suppressing VOCs (Raza et al., 2020). In our study, the underlying mechanisms behind VOC-mediated pathogen suppression and plant-growth promotion seemed to be different as most of the VOCs showed pathogensuppression activity and only one VOC, heptadecane, produced by Pseudomonas strains showed both pathogen suppression and plant growth-promoting activity (Fig. S8). Bacteria often upregulate levels of secondary metabolism and turn more antagonistic as a result of interactions between species or by sensing chemical cues from the competitors (Haas and Keel, 2003; Dubuis and Haas, 2007; Garbeva et al., 2011). It is thus possible that in this study, the presence of other bacterial strains promoted antagonism and thus new pathogen-suppressing VOCs were produced because they are also used in interference competition between VOCproducing species (Becker et al., 2012). This effect was found to be prominent in Gram-positive Bacillus strains as eight new VOCs, including five pathogen-suppressing VOCs, were produced at different community richness levels compared to the monocultures. Alternatively, Gram-negative Pseudomonas strains produced only three new VOCs, including one pathogen-suppressing $\mathrm{VOC}$ but in a relatively small amount.

3.5 Linking bacterial community productivity with the production of pathogen suppressing and plant growth-promoting VOCs

We next investigated if and how the production of pathogen suppressing and plant growth-promoting VOCs were linked with bacterial community productivity (growth). The community productivity of Pseudomonas strains showed nonsignificantly increasing trend with the increase in community richness (Fig. $3 \mathrm{~A}$ ) and showed a hump-shaped relationship with both the relative amount and numbers of pathogen suppressing and plant growth-promoting VOCs (Fig. 4A-D). Alternatively, the community productivity of Bacillus strains decreased significantly with an increase in bacterial community richness (Fig. 3B) and showed a hump-shaped relationship with both the relative amount and numbers of pathogen suppressing and plant growth-promoting VOCs (Fig. 4E-H). These results revealed that the bacterial community interactions played an important role in the production of VOCs and 

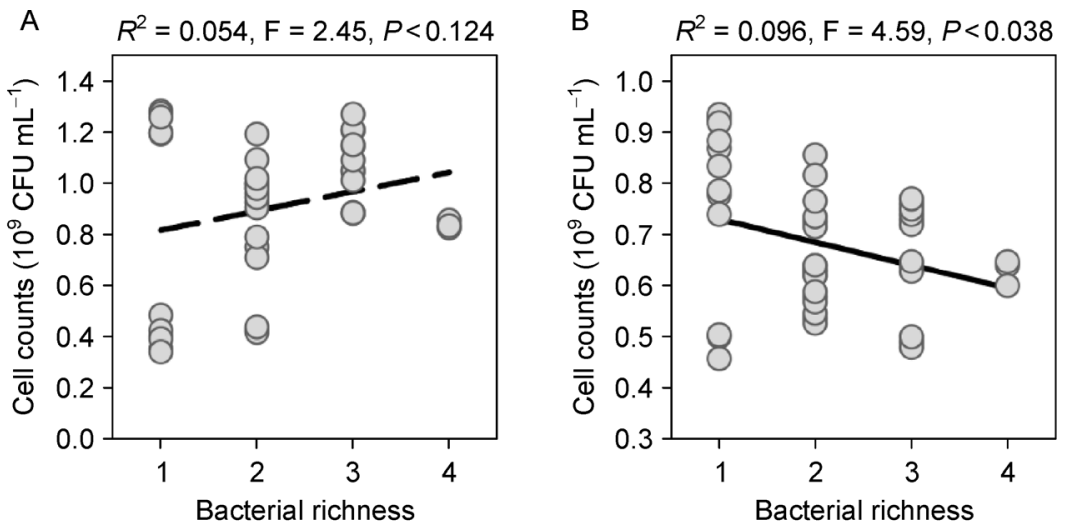

Fig. 3 Effect of bacterial community richness on community productivity (total bacterial abundance). Relationships between Pseudomonas (A) and Bacillus (B) on bacterial community richness and total bacterial community productivity. CFU denotes bacterial cell numbers per $\mathrm{ml}$ in terms of colony forming units. Each observation shows the effect of each replicate in each bacterial monoculture or community.
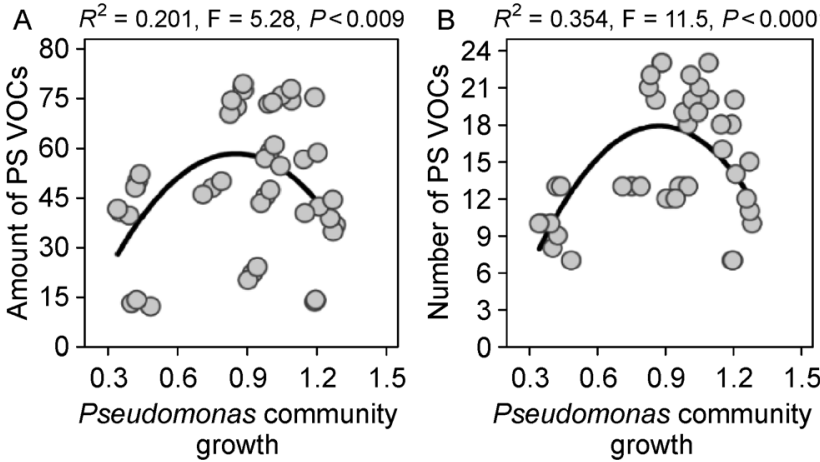

growth

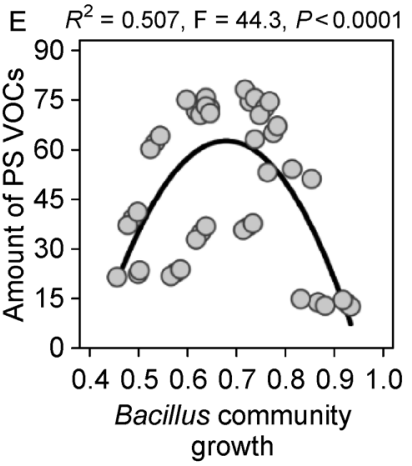

$\mathrm{F} \quad R^{2}=0.325, \mathrm{~F}=20.74, P<0.000$

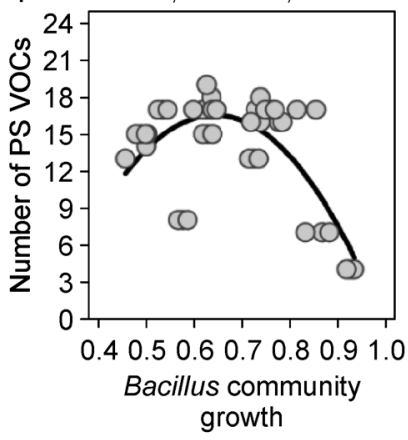

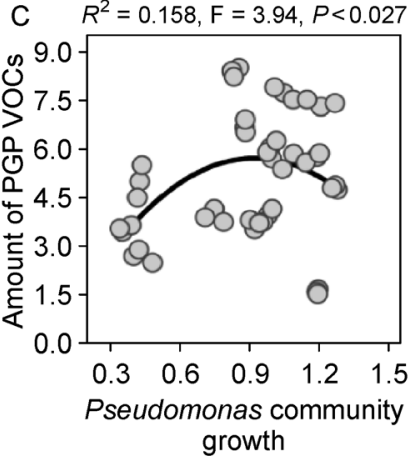

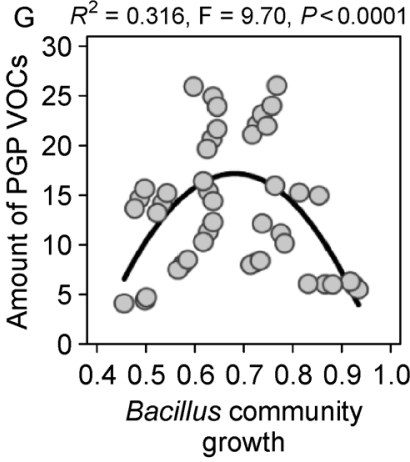

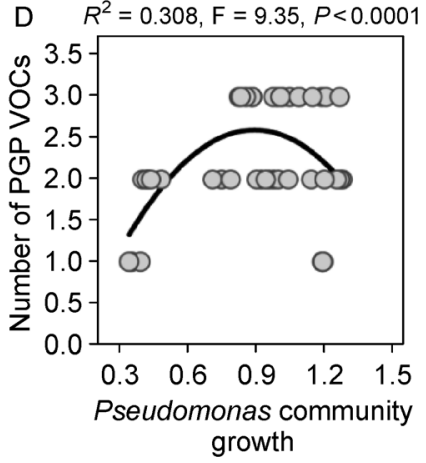

$\mathrm{H} \quad R^{2}=0.223, \mathrm{~F}=6.03, P<0.005$

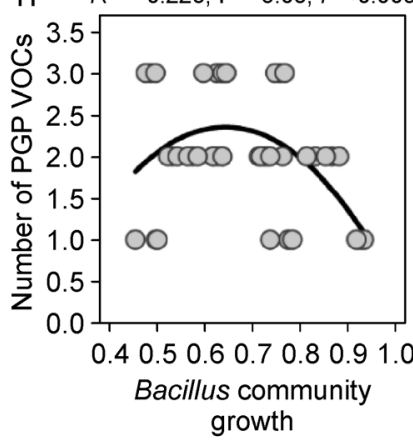

Fig. 4 Interaction of community productivity (growth) at different bacterial richness levels with the relative amount and numbers of VOCs produced by Pseudomonas and Bacillus strains. The top panel shows the interaction of community productivity at different richness levels with the relative amount $(A)$ and numbers $(B)$ of pathogen suppressing (PS) and relative amount (C) and numbers (D) of plant growth-promoting (PGP) VOCs produced by Pseudomonas strains. The bottom panel shows the interaction of community productivity at different richness levels with the relative amount $(E)$ and numbers $(F)$ of $P S$ and relative amount $(G)$ and numbers $(\mathrm{H})$ of plant growth-promoting $(\mathrm{PGP})$ VOCs produced by Bacillus strains.

the maximum production of VOCs was achieved at the intermediate growth of bacterial communities of both Pseudomonas and Bacillus genera. The high and low community productivity reduced the production of pathogen suppressing and plant growth-promoting VOCs. Schulz-Bohm et al. (2015) also reported that both the composition of microbial communities and interactions among community members play an important role in the production of VOCs. Bacterial community productivity is shaped by natural selection in response to resource conditions over their ancestral history (Maitra and Dill, 2015). In this study, Pseudomonas strains might maintain community productivity because of their 
versatile metabolism (Rojo, 2010) compared to Bacillus strains, which showed a decrease in community productivity with the increase in community richness. This decrease in community productivity might trigger the antagonistic interactions because of interference competition among Bacillus strains as is evident from the production of eight new VOCs at different community richness levels, including five pathogensuppression VOCs, while this antagonism was unsubstantial in the case of Pseudomonas strains.

\section{Conclusion}

In heterogeneous environments like the soil, different bacterial strains have different ecological characteristics, and the bacterial cells are patterned by their interactions with neighboring cells and the abiotic environment (Liu et al., 2017). Here we evaluated how intra-species interactions of each Gram-positive and Gram-negative genus mediate the production and functioning of VOCs. The results clearly showed that the bacterial interactions within multispecies communities affect the amount and type of produced VOC. This effect not only includes the blend of VOCs from all strains in a community but also the production of new VOCs. In turn, this changes the VOC-mediated functioning in terms of pathogen suppression and plant-growth promotion. However, the emergence of new VOCs depends on the interactions within species. While both genera Bacillus and Pseudomonas showed similar results for VOC-mediated pathogen suppression and plant-growth promotion, the intra-species interactions revealed some differences. Gram-positive Bacillus strains showed a decrease in community productivity and triggered antagonism, which resulted in the emergence of new pathogen-suppressing VOCs. Together, this study suggests that both bacterial identity and diversity need to be considered for understanding the VOC-mediated activity of bacterial communities. From the applied perspective, our study suggests that VOC-mediated functions could be employed to manipulate the microbiome composition of the rhizosphere to simultaneously improve multiple ecosystem functions including pathogen suppression and plant growth.

\section{Acknowledgment}

The work is supported by the National Natural Science Foundation of China (Grant numbers 31601835, 41671248, 41671256); National Key Basic Research Program of China (Grant numbers 2015CB150503, 2015CB150500), the Fundamental Research Funds for the Central Universities (Grant numbers KYT201802, KJQN201745), and Jiangsu Science and Technology Department (Grant numbers BK20171373, BK20170085).

\section{References}

Becker, J., Eisenhauer, N., Scheu, S., Jousset, A., 2012. Increasing antagonistic interactions cause bacterial communities to collapse at high diversity. Ecology Letters 15, 468-474.

Bell, T., Lilley, A.K., Hector, A., Schmid, B., King, L., Newman, J.A., 2009. A linear model method for biodiversity-ecosystem functioning experiments. American Naturalist 174, 836-849.

Dubuis, C., Haas, D., 2007. Cross-species GacA-controlled induction of antibiosis in Pseudomonads. Applied and Environmental Microbiology 73, 650-654.

Garbeva, P., Hol, W.H.G., Termorshuizen, A.J., Kowalchuk, G.A., de Boer, W., 2011. Fungistasis and general soil biostasis-a new synthesis. Soil Biology \& Biochemistry 43, 469-477.

Gu, Y.Q., Mo, M.H., Zhou, J.P., Zou, C.S., Zhang, K.Q., 2007. Evaluation and identification of potential organic nematicidal volatiles from soil bacteria. Soil Biology \& Biochemistry 39 , 2567-2575.

Haas, D., Keel, C., 2003. Regulation of antibiotic production in rootcolonizing Peudomonas spp. and relevance for biological control of plant disease. Annual Review of Phytopathology 41, 117-153.

Harwood, C.R., Cutting, S.M., 1990. Chemically defined growth media and supplements. In: Harwood, C.R., Cutting, S.M., eds. Molecular Biological Methods for Bacillus. Chichester: Wiley, p. 548

Hazard, C., Kruitbos, L., Davidson, H., Mbow, F.T., Taylor, A.F.S., Johnson, D., 2017. Strain identity of the ectomycorrhizal fungus Laccaria bicolor is more important than richness in regulating plant and fungal performance under nutrient rich conditions. Frontiers in Microbiology 8, 1874.

Hu, J., Wei, Z., Weidner, S., Friman, V.P., Xu, Y.C., Shen, Q.R., Jousset, A., 2017. Probiotic Pseudomonas communities enhance plant growth and nutrient assimilation via diversity-mediated ecosystem functioning. Soil Biology \& Biochemistry 1113, 122 129.

Jousset, A., Becker, J., Chatterjee, S., Karlovsky, P., Scheu, S., Eisenhauer, N., 2014. Biodiversity and species identity shape the antifungal activity of bacterial communities. Ecology 95, 11841190.

Jousset, A., Schmid, B., Scheu, S., Eisenhauer, N., 2011. Genotypic richness and dissimilarity opposingly affect ecosystem functioning. Ecology Letters 14, 537-545.

Jurburg, S.D., Salles, J.F., 2015. Functional redundancy and ecosystem function - the soil microbiota as a case study. In: Lo, Y.H., Blanco, J.A., Roy, S., eds. Biodiversity Ecosystems-Linking Structure and Function. Rijeka: InTech, pp. 29-49.

Kanchiswamy, C.N., Malnoy, M., Maffei, M.E., 2015. Chemical diversity of microbial volatiles and their potential for plant growth and productivity. Frontiers of Plant Science 6, 151.

Lazazzara, V., Perazzolli, M., Pertot, I., Biasioli, F., Puopolo, G., Cappellin, L., 2017. Growth media affect the volatilome and antimicrobial activity against Phytophthora infestans in four Lysobacter type strains. Microbiological Research 201, 52-62.

Leff, J.W., Fierer, N., 2008. Volatile organic compound (VOC) emissions from soil and litter samples. Soil Biology \& Biochemistry 40, 1629-1636.

Liu, A., Archer, A.M., Biggs, M.B., Papin, J.A., 2017. Growth-altering microbial interactions are responsive to chemical context. PLoS One 12, e0164919.

Loreau, M., Hector, A., 2001. Partitioning selection and complementarity in biodiversity experiments. Nature $412,72-76$. 
Maitra, A., Dill, K.A., 2015. Bacterial growth laws reflect the evolutionary importance of energy efficiency. Proceedings of the National Academy of Sciences of the United States of America 112, 406- 411.

Mendes, L.W., Brossi, M.J.L., Kuramae, E.E., Tsai, S.M., 2015. Landuse system shapes soil bacterial communities in Southeastern Amazon region. Applied Soil Ecology 95, 151-160.

Raza, W., Wang, J., Jousset, A., Friman, V.P., Mei, X., Wang, S., Wei, Z., Shen, Q., 2020. Bacterial community richness shifts the balance between volatile organic compound-mediated microbepathogen and microbe-plant interactions. Proceedings. Biological Sciences 287, 20200403.

Raza, W., Wang, J., Wu, Y., Ling, N., Wei, Z., Huang, Q., Shen, Q.R., 2016. Effects of volatile organic compounds produced by Bacillus amyloliquefaciens on the growth and virulence traits of tomato bacterial wilt pathogen Ralstonia solanacearum. Applied Microbiology and Biotechnology 100, 7639-7650.

Raza, W., Yuan, J., Ling, N., Huang, Q., Shen, Q., 2015. Production of volatile organic compounds by an antagonistic strain Paenibacillus polymyxa WR-2 in the presence of root exudates and organic fertilizer and their antifungal activity against Fusarium oxysporum $\mathrm{f}$. sp. niveum. Biological Control 80, 89-95.

Rojo, F., 2010. Carbon catabolite repression in Pseudomonas: optimizing metabolic versatility and interactions with the environ- ment. FEMS Microbiology Reviews 34, 658-684.

Schmidt, R., Cordovez, V., de Boer, W., Raaijmakers, J., Garbeva, P., 2015. Volatile affairs in microbial interactions. ISME Journal 9, 2329-2335.

Schulz, S., Dickschat, J.S., 2007. Bacterial volatiles: the smell of small organisms. Natural Product Reports 24, 814-842.

Schulz-Bohm, K., Zweers, H., de Boer, W., Garbeva, P., 2015. A fragrant neighborhood: volatile mediated bacterial interactions in soil. Frontiers in Microbiology 6, 1212.

Tahir, H.A., Gu, Q., Wu, H., Raza, W., Hanif, A., Wu, L., Colman, M.V., Gao, X., 2017. Plant growth-promotion by volatile organic compounds produced by Bacillus subtilis SYST2. Frontiers in Microbiology 8, 171.

Tyc, O., Zweers, H., de Boer, W., Garbeva, P., 2015. Volatiles in interspecific bacterial interactions. Frontiers in Microbiology 6, 1412.

Wagg, C., Jansa, J., Schmid, B., van der Heijden, M.G.A., 2011. Belowground biodiversity effects of plant symbionts support aboveground productivity. Ecology Letters 14, 1001-1009.

Wei, Z., Yang, X.M., Yin, S.X., Shen, Q., Ran, W., Xu, Y., 2011. Efficacy of Bacillus-fortified organic fertiliser in controlling bacterial wilt of tomato in the field. Applied Soil Ecology 48, 152-159.

Westhoff, S., van Wezel, G.P., Rozen, D.E., 2017. Distancedependent danger responses in bacteria. Current Opinion in Microbiology 36, 95-101. 